\title{
Hippocampal network reorganization underlies the formation of a temporal association memory
}

Mohsin S. Ahmed ${ }^{1,2, *}$, James B. Priestley ${ }^{2,3,4,}{ }^{*}$, Angel Castro ${ }^{1,2}$, Fabio Stefanini ${ }^{3,4}$, Elizabeth M. Balough ${ }^{2,3}$, Erin Lavoie ${ }^{1,2}$, Luca Mazzucato ${ }^{2,4,5,6}$, Stefano Fusi ${ }^{2,4,5}$, Attila Losonczy ${ }^{2,5 \dagger}$

1 Department of Psychiatry

2 Department of Neuroscience

3 Doctoral Program in Neurobiology and Behavior

${ }^{4}$ Center for Theoretical Neuroscience

5 Mortimer B. Zuckerman Mind Brain Behavior Institute

Columbia University, New York, NY 10027 USA

${ }^{6}$ Departments of Mathematics and Biology

University of Oregon, Eugene, OR 97403 USA

* These authors contributed equally to this work

$\dagger$ To whom correspondence should be addressed: al2856@columbia.edu 


\section{Abstract}

Episodic memory requires linking events in time, a function dependent on the hippocampus. In 2 "trace" fear conditioning, animals learn to associate a neutral cue with an aversive stimulus despite 3 their separation in time by a delay period on the order of tens of seconds. But how this temporal 4 association forms remains unclear. Here we use 2-photon calcium imaging to track neural 5 population dynamics over the complete time-course of learning and show that, in contrast to 6 previous theories, the hippocampus does not generate persistent activity to bridge the time delay. 7 Instead, learning is concomitant with broad changes in the active neural population in CA1. While 8 neural responses were highly stochastic in time, cue identity could be reliably read out from 9 population activity rates over longer timescales after learning. These results question the ubiquity 10 of neural sequences during temporal association learning, and suggest that trace fear conditioning 11 relies on mechanisms that differ from persistent activity accounts of working memory.

\section{Introduction}

Episodic memory recapitulates the sequential structure of events that unfold in space and time Eichenbaum, 2017]. In the brain, the hippocampal network is critical for binding the representations of discontiguous events Kitamura et al., 2015, Eichenbaum, 2017]. These findings 16 are corroborated by recent evidence that the hippocampus generates sequences of neural activity $\quad 17$ that bridge the gap between sensory experiences (Pastalkova et al., 2008, MacDonald et al., 2011, 18 Wang et al., 2015, Robinson et al., 2017, and that these dynamics are critical for memory Wang 19 et al., 2015, Robinson et al., 2017]. However, it remains a longstanding challenge to track how 20 hippocampal coding is modified over the course of episodic learning that requires the association of ${ }_{21}$ events in time.

Pavlovian fear conditioning provides an attractive framework to study the neuronal correlates ${ }_{23}$ and mechanisms of associative learning in the brain Letzkus et al., 2015, Grundemann and Luthi, 24 2015, Maddox et al., 2019, Grewe et al., 2017. Classical "trace" fear conditioning (tFC) has long 25 been used as a model behavior in the hippocampal literature for studying temporal association 26 
learning [Raybuck and Lattal, 2014, Kitamura et al., 2015. In this paradigm, subjects learn that a ${ }_{27}$ neutral conditioned stimulus (CS) predicts an aversive, unconditioned stimulus (US), which follows 28 the CS by a considerable time delay (the "trace" period). Circuitry within the dorsal hippocampus 29 is required for forming these memories at trace intervals on the scale of tens of seconds Raybuck 30 and Lattal, 2014, Huerta et al., 2000, Quinn et al., 2005, Fendt et al., 2005, Chowdhury et al., 2005, 31 Sellami et al., 2017. Further, silencing activity in CA1, the output node of the hippocampus, 32 during the trace period itself is sufficient to disrupt the temporal binding of the CS and US in 33 memory Sellami et al., 2017]. While these experiments pinpoint a role for hippocampal activity in 34 forming trace fear memories, the underlying neural dynamics remain unresolved. Importantly, tFC 35 precludes a simple Hebbian association of CS and US selective neural assemblies, due to the 36 non-overlapping presentation of the stimuli.

Previous work has proposed that persistent activity mechanisms enable the hippocampus to connect representations of events in time, bridging time gaps on the order of tens of seconds. In particular, theories suggest that representation of the neutral CS and aversive US are linked through the generation of stereotyped, sequential activity in CA1 Kitamura et al., 2015, Sellami ${ }_{41}$ et al., 2017], even when animals are immobilized [MacDonald et al., 2013]. Alternatively, hippocampal activity could generate a sustained response to the CS in order to maintain a static $\quad 43$ representation of the sensory cue in working memory over the trace interval, such as in attractor $\quad 44$ models of neocortical delay period activity Amit and Brunel, 1997, Barak and Tsodyks, 2014, 45 Takehara-Nishiuchi and McNaughton, 2008 and recently in the human hippocampus Kaminski et al. 2017 during working memory tasks. However, these hypotheses of persistent activity remain 47 to be tested during trace fear learning.

Here we leveraged 2-photon microscopy and functional calcium imaging to record the dynamics 49 of CA1 neural populations longitudinally as animals underwent trace fear learning, in order to 50 resolve the underlying patterns of network activity and their modifications in response to learning. 51 Our findings show that persistent activity does not manifest during this paradigm, incongruous 52 with sequence or attractor models of temporal association learning. Instead, learning instigated 53 broad changes in network activity and the emergence of a sparse and temporally stochastic code for 54 CS identities that was absent prior to conditioning. These findings suggest the role of the 
hippocampus in trace conditioning may be fundamentally different from learning that requires

\section{Results}

We previously developed a head-fixed variant of an auditory trace fear conditioning paradigm

In order to investigate the underlying network dynamics in the hippocampus that accompanied 71 trace fear learning, we selectively expressed the fluorescent calcium indicator GCaMP6f in CA1 72 pyramidal neurons (Fig. 1B B) via injection of a Cre-dependent AAV in CaMKII $\alpha$-Cre mice 
A
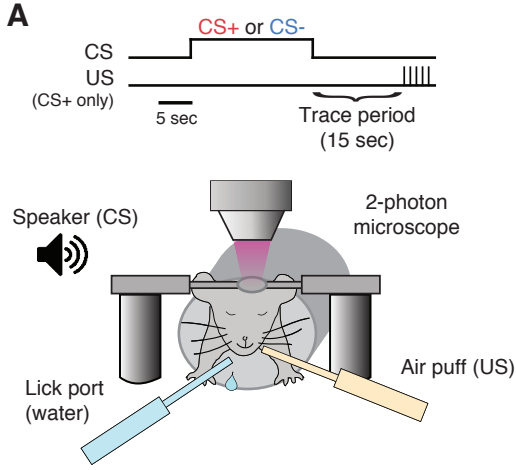

B

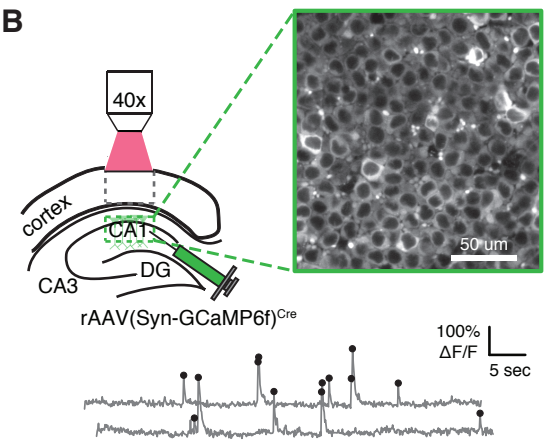

C

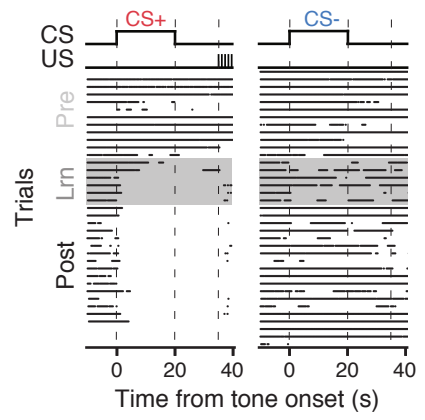

D

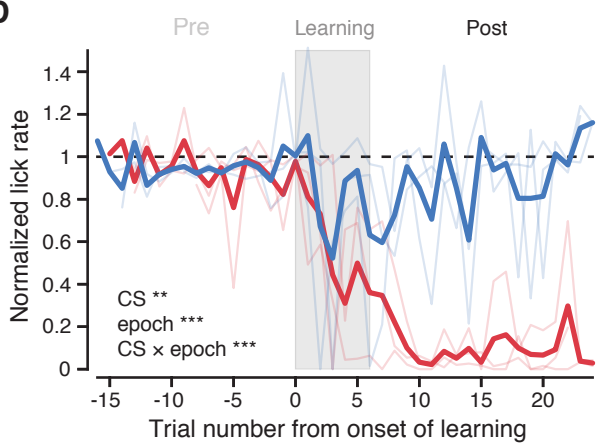

Figure 1. 2-photon functional imaging of CA1 pyramidal neurons during differential trace fear conditioning. A: Schematic of the differential trace fear conditioning $(\mathrm{tFC})$ paradigm. A head-fixed mouse is immobilized and on each trial exposed to an auditory cue (CS+ or CS-) for 20 seconds. This is followed by a 15-second stimulus-free 'trace' period, after which the US is triggered (CS+ trials only). Air-puffs are used as the US and lick suppression as a measure of learned fear. Operant water rewards are available throughout all trials. B: Top, schematic of in vivo imaging preparation with example 2-photon field of view in dorsal hippocampal area CA1. Bottom, calcium traces (grey) and inferred event times (black) from an example neuron. C: Behavioral data for an example mouse over the complete tFC paradigm. Each row is a trial, where dots indicate licks. CSs are first presented without US pairing ('Pre-Learning' epoch). Mice then rapidly learn to discriminate CSs and associate the CS+ with the US over the first 6 paired trials ('Learning' epoch), after which we continue to collect additional trials ('Post-Learning' epoch). D: Summary of behavioral dataset. We compute a normalized lick rate for each trial by dividing the lick rate during the CS tone $(0-20 \mathrm{sec})$ period by the lick rate in the pre-CS $(-10$ to $0 \mathrm{sec})$ period. Bold lines are averages across mice. Thin lines show individual mice $(n=3$ mice, linear mixed-effects model with fixed effects of CS and learning epoch, with mouse as random effect, main effect significance shown in inset, post hoc models fit to each epoch separately with fixed effects of CS and trial number, Pre-Learning: no significant effects, Learning: effect of trial number $(* * *)$ and CS $\times$ trial number $\left({ }^{*}\right)$, Post-Learning, effect of CS $\left({ }^{* * *}\right)$, Wald $\chi^{2}$ test). ${ }^{*} p<0.05,{ }^{* *} p<0.01,{ }^{* * *} p<0.001$

with continued US reinforcement to avoid extinction of learned fear. Mice readily discriminated between the two cues throughout Post-Learning, as they suppressed licking consistently on CS+ 84 trials but not CS- trials, where the air-puff was never presented (Fig. 1C,D).

Fluorescence imaging data from each trial was motion corrected Kaifosh et al., 2014], and ROI 
were each active on at least 4 trials, which were used for subsequent analyses (Fig. 2A). Neural 90 activity spanned all trial periods during the task both Pre- and Post-Learning, with a clear increase 91 in neural activity following learning (population average event rate from 0-35 sec (events/sec): Pre: 92 0.039, Post: $0.057, p<1.67 \mathrm{e}-10$, signed rank test) and a large population response to the US (Fig. 93 2 A).

We first asked whether the hippocampus generated a consistent temporal code during each trial 95 to connect the CS and US representations Sellami et al., 2017, Kitamura et al., 2015]. While 96 ordering population activity by the latency of neurons' peak firing rates naturally lends the 97 appearance of a sequence that spans the trial period (Fig. 22A), this ordering must be consistent 98 across trials in order to be useful for computation. We approached this question through decoding, 99 as the presence of sequential dynamics such as "time cells" [MacDonald et al., 2011] should allow us 100 to decode the passage of time from the neural data Bakhurin et al., 2017, Robinson et al., 2017, ${ }_{101}$ Cueva et al. 2019]. We used an ensemble of linear classifiers trained to discriminate the population 102 activity between every pair of time points Bakhurin et al., 2017, Cueva et al., 2019] in the tone and 103 trace periods of the trial (0-35 sec, $2.5 \mathrm{sec}$ bins). To illustrate the idea behind this analysis, we can 104 summarize the activity of the network at each point in time as a point in a high dimensional neural 105 state space, where the axes in this space corresponds to the activity rate of each neuron 106 (schematized in Fig. 2B). The state of the network at each point in time during a trial traces out a 107 path of points in neural state space. If the neural dynamics continually evolve in time (e.g. time 108 cell sequences), then the neural state at one point in time $(t)$ should be different from the states $\quad 109$ that occur at points further away in time $(t+\Delta t)$, reflecting the recruitment of different neurons at 110 each point in the sequence. If these dynamics are reliable across many trials, we should be able to 111 train a linear decoder to accurately classify whether data came from one time point or the other, by 112 finding the hyperplane that maximally separates data from time $t$ and $t+\Delta t$ in the neural state $\quad 113$ space. By extending this analysis to compare all possible pairs of time points (i.e. for all possible ${ }_{114}$ $\Delta t \mathrm{~s}$ ), we can identify moments during the task that exhibit reliable temporal dynamics across trials 115 Bakhurin et al., 2017, Cueva et al., 2019. We note however that the ability to decode time is not ${ }_{116}$ an exclusive feature of neural sequences, but a signature of any consistent dynamical trajectory $\quad 117$ where the neural states become sufficiently decorrelated in time (e.g. consider a population of 118 
"ramping" cells whose firing ranges change monotonically as a function of time [Cueva et al., 2019], 119 or a chaotic trajectory in the activity space Buonomano and Maass, 2009]). Accordingly, our 120 analysis first addresses this broader question of whether any temporal coding arises during the task, 121 without a priori assumptions on its parametric form.

We used these classifiers to assess whether neural activity was linearly separable between each 123 pair of time points in the tone and trace periods of the task. Fig. $2 \mathrm{C}$ shows the results of this analysis for an example mouse during CS+ Post-Learning trials. The cross-validated decoding accuracy was no better than chance for most pairwise classifiers, suggesting that either the pattern
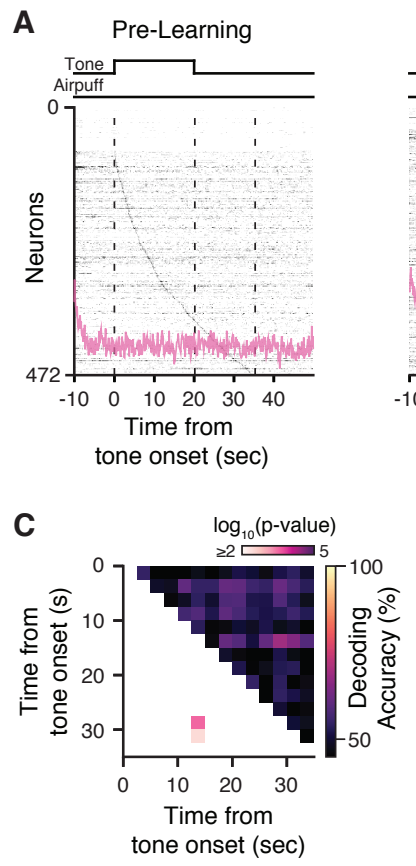

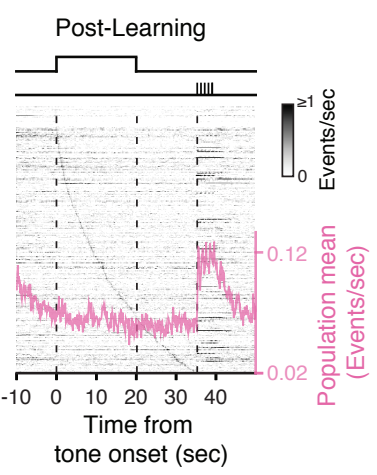

D

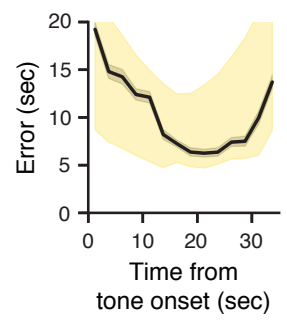

B
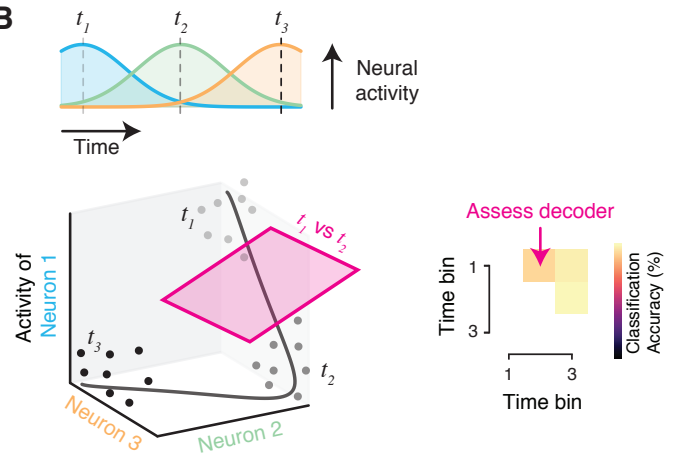

E

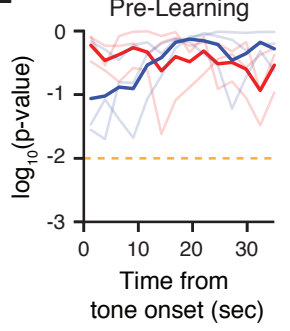

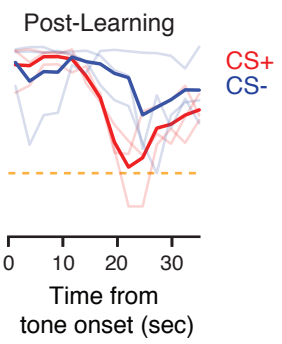

Figure 2. Temporal dynamics of CA1 population activity during trace fear conditioning. A: Summary of neural activity during Pre- and Post-Learning trials. For each epoch, activity is trial-averaged and neurons are sorted by the latency of their peak firing rate during the CS and trace periods $(0-35 \mathrm{sec})$ during. The population average event rate is overlaid. B: Schematic of time decoding analysis. Top: trial-averaged tuning curves of a hypothetical sequence of time cells. Bottom: state space representation of the neural data. Dots indicate the neural state on single trials at three time points in the task. Right: A separate linear classifier (support vector machine; SVM) was trained to discriminate between population activity from every pair of time points in the task. C: Matrix of classifiers for an example mouse during Post-Learning CS+ trials. The upper triangle reports the cross-validated accuracy of classifiers trained to discriminate between the corresponding pair of time points. The lower triangle reports the p-value relative to a shuffle distribution. Most pairwise classifiers perform at chance level. D: Time prediction performance for the example shown in $\mathbf{C}$. For each time bin in a test trial, the population activity is assessed by all classifiers, whose outputs are combined via a voting procedure to determine the decoded time. Decoding accuracy is assessed as the absolute error between real and predicted time. Black: cross-validated average and bootstrapped $95 \%$ confidence interval for time decoding error. Yellow shading: $95 \%$ bounds of the null distribution. Decoding error is within chance levels throughout the trial. E: Summary of time decoding significance relative to the null distribution during Pre-Learning (left) and Post-Learning (right) trials. 
of neural activity remains relatively constant throughout the tone and trace periods, or the

dynamics are not consistent across trials. As an additional test of temporal coding, we can combine the output of the classifier ensemble to predict the time bin label of individual activity vectors from 129 held out test trials ("one-vs-one" multi-class prediction, Bakhurin et al., 2017, Cueva et al., 2019]). 130 For each time bin in a test trial, the neural activity at that time is provided as input to all pairwise ${ }_{131}$ classifiers, whose binary decisions are combined via a voting procedure to determine the predicted 132 time bin label of the data. Despite combining the information learned by all classifiers, time 133 decoding accuracy did not exceed chance-level performance (Fig. 2D). We did not find evidence of 134 significant temporal coding during either Pre- or Post-Learning trials (Fig. 2E); we did observe 135 some trend toward significance during Post-Learning, which may reflect broader timescale differences in population activity during the CS and trace periods (see Fig. 4). Overall, these ${ }_{137}$ results suggest that CA1 neural activity sequences are not a reliable phenomenon during trace fear 138 memory.

Since animals learned the association within the first few CS+/US pairings (Fig. 1C,D), we ${ }_{140}$ separately assessed whether any sequential dynamics might have rapidly and transiently emerged 141 during the initial "Learning" trials. Due to the lower number of trials available, decoding was not 142 possible, and so we computed a population sequence score by computing the rank correlation 143 between the firing sequence of neurons across Learning trials (Fig. S2). However we found that ${ }_{144}$ neural activity did not organize into any reliable temporal patterns during these initial CS-US 145 pairing trials. These data suggest that consistent temporal coding is not a dominant network 146 phenomenon during trace fear conditioning, and so stereotyped sequential activity is unlikely to $\quad{ }_{147}$ bridge the gap between CS and US presentations during the initial learning phase.

Our time decoding analyses indicated that most periods in time during the task were indistinguishable, which suggests that the network state during each trial may be relatively static. 150 We considered an alternative hypothesis consistent with static activity, where CS information is 151 maintained by persistent activation of a subgroup of hippocampal neurons Kaminski et al., 2017, 152 as in attractor models of neocortical networks suggested to underlie working memory Amit and 153 Brunel, 1997, Barak and Tsodyks, 2014, Takehara-Nishiuchi and McNaughton, 2008]. Under this ${ }_{154}$ scenario, the population dynamics would not evolve in time but discretely shift to a static state $\quad 155$ 

state space by a hyperplane (Fig. 3A).

For each time bin, we assessed whether we could accurately decode identity of the CS, separately 161 analyzing Pre- and Post-Learning trials. Fig. 3B show the results of this analysis for an example 162 Post-Learning time bin, reported as the percentage of correctly decoded trials and compared to a 163 null distribution generated by shuffling the trial type identities. Our choice of linear classifiers also 164 allowed us to obtain an intuitive measure of the importance of each neuron to the decoder's 165 decisions, by examining the weights of each neuron along a vector orthogonal to the separating 166 hyperplane (w, Fig. 3A,C, Stefanini et al. 2019]). Examining these results across time bins, we 167 found that we were unable to decode the CS identity during Pre-Learning or Post-Learning trials at 168 any point prior to the delivery of the US (Fig. 3D). We verified that this was not due to our choice 169 of decoder; CS decoding conclusions were unchanged when we used a Bayesian approach (Fig. S3). 170 Similar methods have been used previously during appetitive trace conditioning paradigms in 171 monkeys, where the CS identity and US prediction could be accurately decoded from the

A

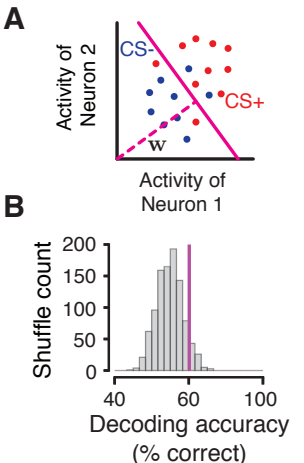

C

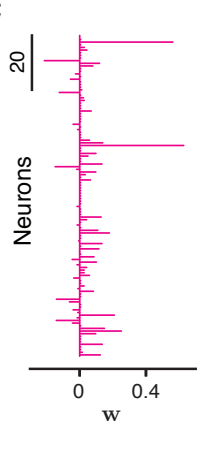

D

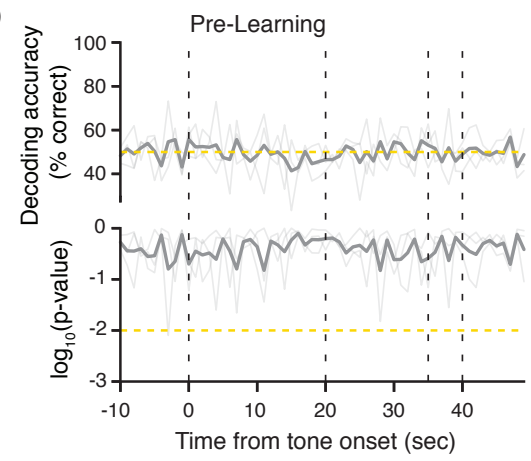

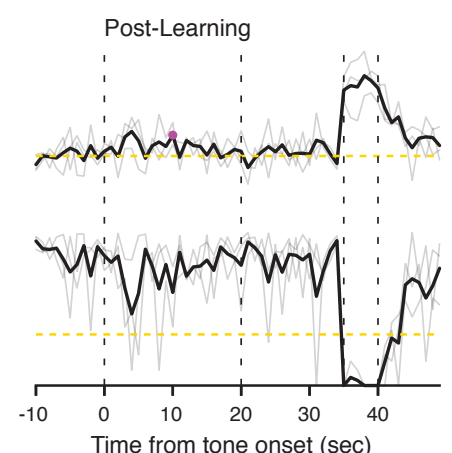

Figure 3. Stimulus identity is not persistently encoded in CA1. A: Schematic of CS decoding analysis. A separate classifier was trained to discriminate between CS+ vs CS- trials using population activity at each time point during the task (1 sec bins). B: Example CS decoder performance during Post-Learning. Purple line: average cross-validated decoding accuracy. Grey histogram: accuracy distribution obtained under surrogate datasets with shuffled trial labels. C: Population decoder weights for the classifier shown in B (averaged over cross-validation folds). D: CS decoding accuracy during Pre-Learning (left) and Post-Learning (right) trials, reported as \% accuracy (top) and p-value relative to the null distributions (bottom). Instantaneous CS decoding accuracy is within chance levels throughout the trial, except during the time of air-puff delivery during Post-Learning trials. The example shown in $\mathbf{B}$ is marked in purple. 
moment-to-moment population activity in the amygdala and prefrontal cortex [Saez et al., 2015]. 173 However, in these experiments the trace period was an order of magnitude shorter than in our fear 174 learning paradigm. These results indicate that information about the CS identity does not appear 175 to be maintained in the moment-to-moment activity of CA1 pyramidal cell populations. Relatedly, 176 activity was not robustly tied to instantaneous licking behavior, which differed markedly between 177 cues during Post-Learning trials (Fig. 1D). CS decoding accuracy was generally high during 178 US-delivery in Post-Learning trials, consistent with the clear population response to the air-puff 179 (Fig. 2A). Though still within chance-level, there was a visible increase in the variability of 180 classifiers' performance during the tone delivery in Post-Learning trials. This suggested to us that 181 there may be cue-selective responses in the population that appeared with variable timing across $\quad{ }_{182}$ trials, and so they could not be reliably decoded at more granular time resolutions.

We tested the hypothesis that neural activity levels were predictive at longer timescales, first by 184 attempting to decode the CS identity from the average activity rate across the CS and trace 185 periods of each trial (Fig. 4A). This analysis is identical to that outlined in Fig. 3, except we ${ }_{186}$ collapsed the activity into a single time bin by averaging each neuron's activity trace from 0-35 sec. 187 Surprisingly, we found that at this timescale, we could significantly decode the CS identity from the 188 population activity rates. Decoding accuracy exceeded chance performance only during 189 Post-Learning trials, congruent with a change in network organization following learning.

As external sensory information about the stimulus is only available during the 20 sec CS period, 191 we next asked whether average activity rates during other trial periods were still predictive of the 192 cue identity, and how those activity patterns compared to those present during the tone 193 presentation. To address this, we constructed a cross-time period decoding matrix, where we 194 trained decoders to predict the CS identity using the average activity in a given time block and 195 tested their performance on the activity from all trial time blocks (Fig. 4B). Values along the 196 diagonal of the matrix gauge how reliably activity during each time block predicts the cue, while ${ }_{197}$ off-diagonal entries assess how decoders generalize to other trial periods. As expected from Fig. 44, 198 decoding during Pre-Learning trials was at chance level for all conditions. During Post-Learning, 199 significant decoding accuracy was observed in all time blocks starting from the tone onset (Fig. 4B). 200 Additionally, decoders showed significant generalization between the CS and trace periods, 201 
A
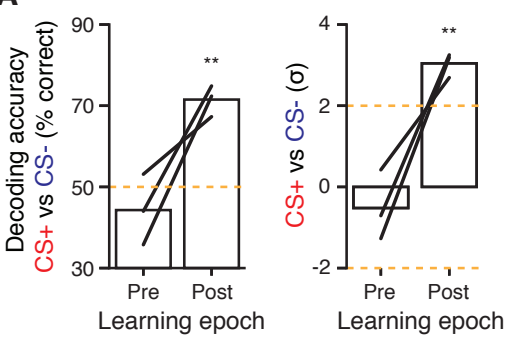

C
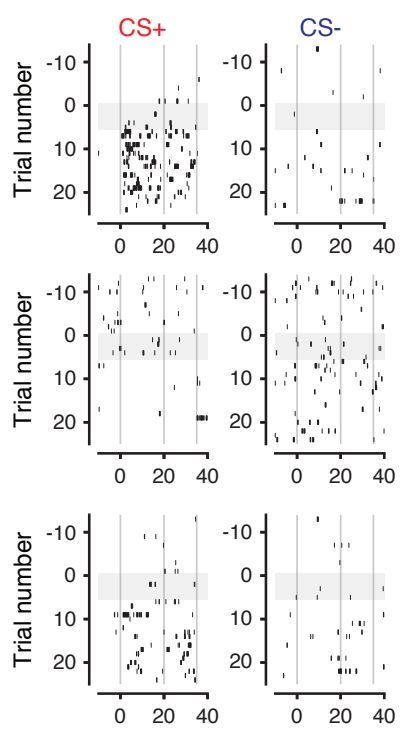

Time from tone onset (sec)
B

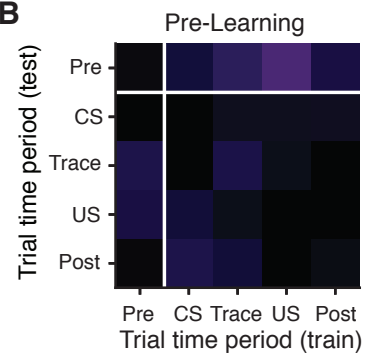

D
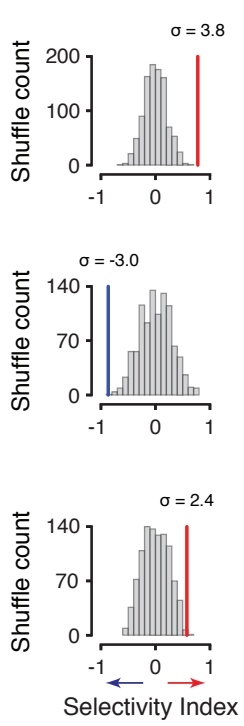

E

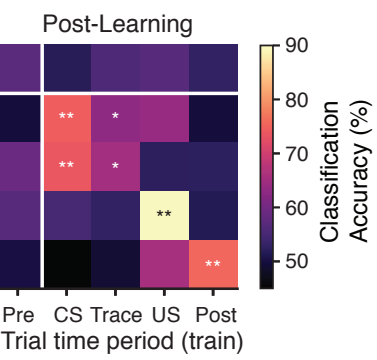

Pre-Learning
Post-Learning
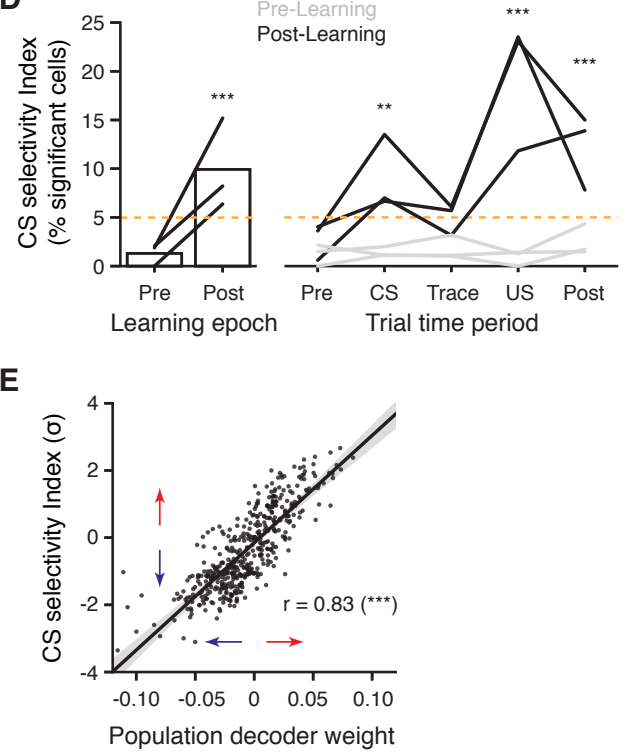

Figure 4. CS identity is predicted by CA1 activity rates on longer timescales. A: CS decoding accuracy for classifiers trained on the average activity within each trial's CS and trace period. Left: \% accuracy, right: z-normalized relative to null distributions calculated as in Fig. 3 Each line is the average cross-validated results from one mouse. B: Decoding CS identity from average activity in each trial time period. Decoders are trained and tested across each possible pair of time periods. In $\mathbf{A}$ and $\mathbf{B}$, asterisks indicate significant p-values relative to shuffle distributions, averaged across mice. C: Raster plots of 3 simultaneously recorded CS-selective neurons (from average activity across CS and trace period). Right: Post-Learning CS selectivity index for each neuron, with shuffle distribution. D: Percentage of active cells with significant CS selectivity. Left: selectivity computed from average activity across CS and trace periods. Right: selectivity computed separately in each trial time period. Each line is a mouse. P-values indicate significant binomial test against the null hypothesis of $\leq 5 \%$, pooled across mice. E: Regression of Post-Learning CS selectivity index for each neuron with its population decoder weight from A. Neurons with high selectivity are highly weighted by the population decoder (Pearson's correlation). ${ }^{*} p<0.05,{ }^{* *} p<0.01$, $* * * p<0.001$

suggesting that a representation of the tone is maintained in the stimulus-free trace interval, and 
associative learning Grewe et al. 2017.

Our analysis established that stimulus identity could be read out from the population activity 209 during the tone and trace period in a learning-dependent manner, and so we sought to connect 210 these findings to changes in neural activity at the level of individual neurons. While some neurons ${ }^{211}$ exhibited very robust cue preferences following learning (Fig. 4 $\mathrm{C}$, top), these were rare and most ${ }_{212}$ cells showed more graded firing rate changes (Fig. 4 C , bottom). We quantified single neuron tuning 213 via a selectivity index, standardized against a shuffle distribution generated by shuffling trial type 214 identities, and measured the fraction of significantly CS-tuned neurons. Single neuron tuning 215 heavily mirrored the population decoding results, both when computed over the tone and trace 216 periods combined and in individual trial time periods (Fig. 4D). Similar to the drop in decoding ${ }^{217}$ accuracy, the fraction of tuned neurons was lower during the trace period than the CS period for all 218 mice. Overall, neurons' normalized CS selectivity indices were extremely correlated with their ${ }^{219}$ weight in the population decoder (Fig. $4 \mathrm{E}$ ), demonstrating that the decoding analysis most heavily 220 relied on neurons in the population with strong tuning to CS identity.

Finally, we sought to characterize how network structure changed during task learning on a 222 trial-to-trial basis. Across the different learning epochs of the task, the fraction of active neurons in ${ }_{223}$ the population significantly increased (Fig. 5A). In addition to heightened network activity, we 224 asked how the set of active neurons compared across trials. To address this question, we measured 225 the overlap between the set of neurons active during the CS and trace periods between each pair of ${ }_{226}$ trials using the Jaccard similarity index (Fig. 5B). These scores were standardized by a null 227 distribution generated by shuffling the active neuron pool in each trial, in order to control for ${ }_{228}$ differences driven trivially by disparities in the number of active neurons. Fig. $5 \mathrm{C}$ depicts the ${ }_{229}$ trial-by-trial overlap in neural ensembles for an example mouse for the complete time course of the 230 experiment, revealing a marked shift in activity patterns from Pre- to Post-Learning. To summarize 231 these observations, we computed the average ensemble similarity to Pre- or Post-Learning trials for 232 each trial in the experiment (Fig. 5D). These results demonstrate that trace fear learning is ${ }^{233}$ accompanied by a large modification in the active neuronal population, beyond that expected from ${ }^{234}$ the overall increase in network activity following conditioning. Given the established role of 235 hippocampal circuits in contextual memory formation Maren et al., 2013, Urcelay and Miller, 2014, 236 

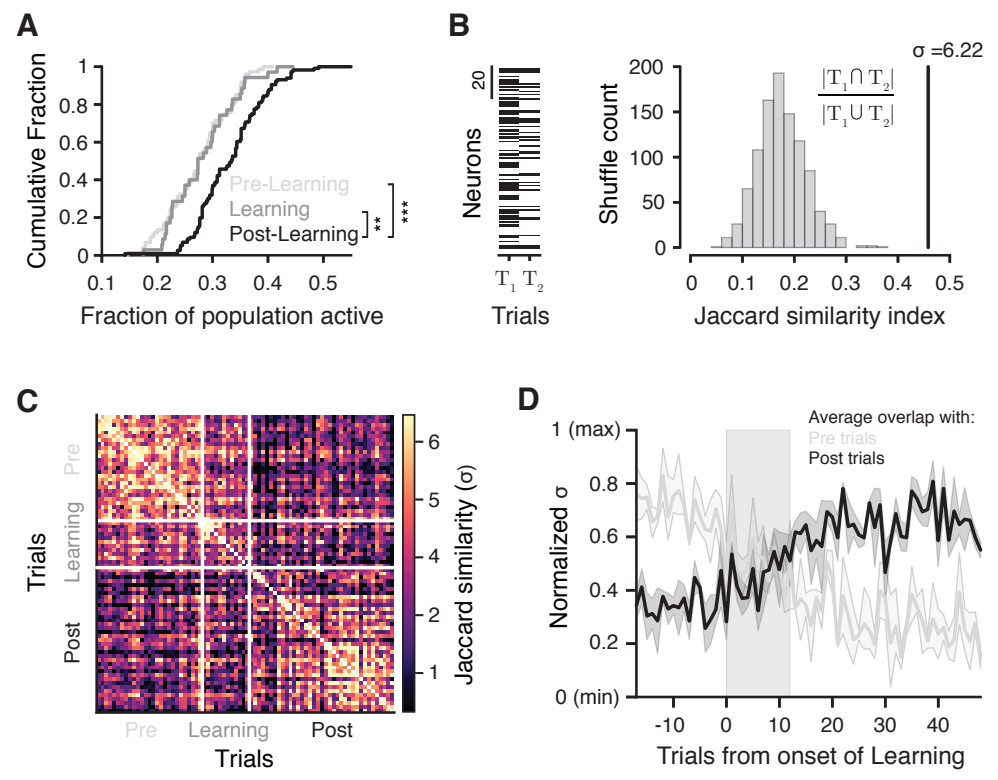

Figure 5. Learning parallels a shift in the active neural population. A. Fraction of neurons active during the tone and trace period, per trial (Kolmogorov-Smirnov test). Trials for both CS cues are pooled. B. Left: example binary activity vectors for two trials. Black indicates the neuron was active on that trial. Right: Jaccard similiarity index for the trial pair shown at left. The observed similarity index is z-scored to a null distribution generated by shuffling the neuron identities on each trial, to control for differences in the number of active neurons. C: Matrix of pairwise z-scored trial similarities for an example mouse. D: Average similarity to Pre- (grey) and Post-Learning (black) trials, for each trial in the experiment (excluding trial self-comparisons). Each mouse is first normalized by its minimum and maximum similarity scores. Learning trials are shaded in Grey. The set of active neurons changes during Learning. ${ }^{*} p<0.05,{ }^{* *} p<0.01,{ }^{* * *} p<0.001$

Fanselow, 2010], this change may also reflect a learning-dependent change in the representation of 237 the broader context, in addition to or independent of the encoding of the individual CS cues.

\section{Discussion}

Here we have implemented a novel experimental framework for deciphering neural coding during non-spatial, temporal associative learning in the hippocampus using chronic cellular imaging. These 241 methods demonstrate that network dynamics during trace fear conditioning are inconsistent with 242 hypotheses of persistent sequential Kitamura et al., 2015, Sellami et al., 2017) or sustained 243 Kaminski et al., 2017] activity in CA1. Rather we find that behavioral learning in CA1 is 244 underpinned by the emergence of a subset of cue-selective neurons with stochastic temporal 245 dynamics across trials. These units encode cue information in a learning-dependent manner. It is 246 plausible that these dynamics may relate to descriptions of hippocampal memory "engram cells", 247 
¿dentified via immediate early gene (IEG) products Liu et al., 2012, Vetere et al., 2017, Tanaka 248 et al., 2018, Rao-Ruiz et al. 2019.

Consistent with this notion, our estimates of significantly CS-selective cells that emerge with 250 learning fall within the range of the 10-20\% of CA1 pyramidal neurons recruited in engrams 251 supporting hippocampal-dependent memory Tayler et al., 2013, Tanaka et al., 2018, Rao-Ruiz 252 et al. 2019. If the sparse subset of CS-selective cells we observe do represent engram cells, then 253 this would further support the notion that gating mechanisms exist to ensure sparsity of encoded 254 engrams, since the size of the engram does not vary with behavioral paradigms, such as 255 immobilized, auditory tFC used here compared with contextual learning in freely moving animals 256 Rao-Ruiz et al. 2019]. It is important to note however that in our data, CS-selectivity at the 257 single cell level manifested along a continuum of firing rate differences between conditions (Fig. 4), 258 and it is unclear how coding differences at these scales would be resolved with IEG-based methods. 259

Our data show that, prior to and following learning, cue information is not actively transmitted 260 by neurons' moment-to-moment firing rates. Neural activity is instead remarkably sparse across 261 time and conditions. The lack of consistent CS coding during the Pre-Learning epoch is consistent 262 with prior evidence that few CA1 pyramidal neurons respond to passive playback of auditory stimuli ${ }_{263}$ Aronov et al. 2017. It is possible that these dynamics also differ according to sensory modality 264 and behavioral states, such as locomotion. In previous studies that report neural sequences in CA1 265 during delay periods Pastalkova et al., 2008, MacDonald et al., 2011, Wang et al., 2015, Robinson 266 et al. 2017, the hippocampal network state was in a regime largely dominated by strong theta 267 pscillations in local field potentials (LFP) and frequent burst firing by pyramidal neurons Buzsáki 268 and Moser, 2013 reminiscent of activity during active behaviors such as spatial exploration. The ${ }_{269}$ dynamics we observe resemble more closely the activity often seen during immobility and awake 270 quiescence, where pyramidal neurons fire only sparsely and in a manner often restricted to 271 population bursts associated with sharp-wave ripple (SWR) LFP events Buzsáki, 2015. 272

We observe sparse and temporally variable activity that nevertheless is predictive of task 273 information when averaged over longer time periods. It is possible then that these dynamics may ${ }^{274}$ arise from stochastic reactivation of memory traces via SWR events. Given the general sparsity of 275 activity during the task and the unreliability of single spike detection with calcium imaging, it is 276 
likely that we have underestimated the task-related activity here. Detection of population burst ${ }^{277}$ pevents is possible at faster imaging speeds and with dense sampling of CA1 populations Malvache 278 et al., 2016, and we speculate that these events may be the underlying cause of the detected CS 279 information following learning. This idea could connect the separate observations that inactivation 280 of the medial entorhinal cortex, a major input to the hippocampus, disrupts both the structure of ${ }_{281}$ SWR events in CA1 Yamamoto and Tonegawa, 2017] and trace fear conditioning Suh et al., 2011. 282

Sparse reactivation of neural assemblies may also suggest a fundamentally different mode of 283 propagating information over time delays during trace fear learning, for example, by storing 284 information transiently in synaptic weights Mongillo et al., 2008, Barak and Tsodyks, 2014. Such ${ }^{285}$ a method could confer a considerable metabolic advantage for maintaining memory traces over long 286 time delays, in contrast to generating persistent activity or neural sequences. Previous theoretical 287 work to this end has focused on short-term plasticity in networks with pre-existing attractor 288 architectures. There, pre-synaptic facilitation among the neurons in a selected attractor drives its 289 reactivation in response to spontaneous input [Mongillo et al., 2008, by out-competing the other 290 attractors. To preserve information about the reactivated attractor on long timescales, they 291 propose a mechanism involving some form of ongoing refreshing activity to maintain synaptic 292 facilitation, which would be incompatible with our observations. As a consequence, the time 293 constant of facilitation limits the lifetime of these memory traces to around the order of a second, 294 which is much shorter than the trace period we considered here. Alternatively, we speculate that 295 coding assemblies may develop through continual Hebbian synaptic potentiation over trials, and 296 that plasticity induced by the most recently presented cue may provide a bias in reactivated ${ }^{297}$ network states on each trial by increasing the depth of the corresponding basins of attraction. A 298 similar scheme has been explicitly modeled in the case of visuo-motor associations Fusi et al., 2007 299 and it is known to require synaptic modifications on multiple timescales Benna and Fusi, 2016. 300 However, it has never been considered in the case of fear conditioning and long time intervals and 301 will be an important direction for future work.

Though differential neural responses to the cues tended to be subtle, we observed an overall 303 marked turnover in the set of active neurons from Pre- to Post-Learning trials that was common for 304 both CS+ and CS- trials. It is possible that this is a broader change in the hippocampal 305 
representation that associates the context with the US itself, or reflects an association with more 306 abstract knowledge of the cue-outcome rules Maren et al., 2013, Urcelay and Miller, 2014, 307 Fanselow, 2010]. Relatedly, past work has shown that memories experienced closely in time may be 308 encoded by overlapping populations of neurons Cai et al. 2016], consistent with our findings of a 309 largely shared neural ensemble between CS trial types in Post-Learning. This linking of distinct but 310 related memories to overlapping populations may occur as a result of transient increases in the 311 excitability of neural subpopulations, which biases allocation into memory engrams Yiu et al., 312 2014, Cai et al., 2016, Rashid et al., 2016. 313

Our findings highlight a hippocampal-dependent learning process that associates events 314 separated in time in the absence of persistent activity. Given that associations in real-world 315 scenarios are often dissociated from emotionally valent outcomes by appreciable time delays 316 Raybuck and Lattal, 2014, our findings have broad implications for models of temporal association 317 learning and circuit dynamics underlying the dysregulation of anxiety and fear in neuropsychiatric 318 $\begin{array}{ll}\text { disorders. } & 319\end{array}$ 


\section{Acknowledgments}

We thank S.A. Siegelbaum, R. Hen, P.D. Balsam, and C.D. Salzman for fruitful discussions. We ${ }_{321}$ thank B.V. Zemelman for viral reagents used in this study. This work was supported by Leon Levy 322 Neuroscience and American Psychiatric Foundation / Genentech, Inc Research Fellowship Awards 323 (to MSA), NIH grants (R25MH086466, T32MH018870, and K08MH113036 to MSA; T32NS064928 324 to JBP; K25DC013557 to LM; R01MH100631, U19NS090583, and R01NS094668 to AL), NSF's 325 NeuroNex program award DBI-1707398 (to FS and SF), the Simons, Grossman, and Gatsby 326 Charitable Foundations (to FS and SF), the Kavli Foundation (to FS, SF, and AL), the Searle 327 Scholars and Human Frontier Science Programs (to AL), and the McKnight Memory and Cognitive 328 Disorders Award (to AL).

\section{Author Contributions}

M.S.A., J.B.P., S.F., and A.L. conceived the project. M.S.A. conducted experiments with inputs 331 from J.B.P., L.M., S.F., and A.L. J.B.P. developed and performed data analyses with L.M., F.S., 332 and S.F., with inputs from M.S.A. and A.L. A.C. designed behavioral hardware. A.C., E.M.B., and 333 E.L. provided technical support. M.S.A, J.B.P., L.M., S.F., and A.L. wrote the manuscript, with 334 input from all authors. 


\section{References}

D. J. Amit and N. Brunel. Model of global spontaneous activity and local structured activity during 339 delay periods in the cerebral cortex. Cerebral cortex (New York, NY: 1991), 7(3):237-252, 1997. 340

D. Aronov, R. Nevers, and D. W. Tank. Mapping of a non-spatial dimension by the 341 hippocampal-entorhinal circuit. Nature, 543(7647):719-722, 2017. ISSN 1476-4687 (Electronic) 342 0028-0836 (Linking). doi: 10.1038/nature21692. URL https://www.ncbi.nlm.nih.gov/pubmed/28358077.

K. I. Bakhurin, V. Goudar, J. L. Shobe, L. D. Claar, D. V. Buonomano, and S. C. Masmanidis. 345 Differential Encoding of Time by Prefrontal and Striatal Network Dynamics. J. Neurosci., 37(4): 346 854-870, dec 2017. ISSN 1529-2401. doi: 10.1523/JNEUROSCI.1789-16.2016. URL 347

O. Barak and M. Tsodyks. Working models of working memory. Curr Opin Neurobiol, 25:20-4, 350 2014. doi: $10.1016 /$ j.conb.2013.10.008. URL

M. K. Benna and S. Fusi. Computational principles of synaptic memory consolidation. Nature ${ }_{353}$ neuroscience, 19(12):1697, 2016.

D. V. Buonomano and W. Maass. State-dependent computations: spatiotemporal processing in 355 cortical networks. Nature Reviews Neuroscience, 10(2):113, 2009.

M. A. Burman, C. A. Simmons, M. Hughes, and L. Lei. Developing and validating trace fear 357 conditioning protocols in c57bl/6 mice. J Neurosci Methods, 222:111-7, 2014. doi:

G. Buzsáki. Hippocampal sharp wave-ripple: A cognitive biomarker for episodic memory and 
D. J. Cai, D. Aharoni, T. Shuman, J. Shobe, J. Biane, W. Song, B. Wei, M. Veshkini, M. La-Vu, ${ }_{364}$ J. Lou, S. E. Flores, I. Kim, Y. Sano, M. Zhou, K. Baumgaertel, A. Lavi, M. Kamata, 365 M. Tuszynski, M. Mayford, P. Golshani, and A. J. Silva. A shared neural ensemble links distinct 366 contextual memories encoded close in time. Nature, 534(7605):115-118, jun 2016. ISSN 367 0028-0836. doi: 10.1038/nature17955. URL http://www.nature.com/articles/nature17955. 368

N. Chowdhury, J. J. Quinn, and M. S. Fanselow. Dorsal hippocampus involvement in trace fear 369 conditioning with long, but not short, trace intervals in mice. Behav. Neurosci., 119(5): 370 1396-1402, 2005. ISSN 1939-0084. doi: 10.1037/0735-7044.119.5.1396. URL 371 http://doi.apa.org/getdoi.cfm?doi=10.1037/0735-7044.119.5.1396. 372

C. J. Cueva, E. Marcos, S. Saez, A. Genovesio, M. Jazayeri, R. Romo, C. D. Salzman, M. N. ${ }^{373}$ Shadlen, and S. Fusi. Low dimensional dynamics for working memory and time encoding. 374 bioRxiv, 2019. doi: 10.1101/504936.

N. B. Danielson, J. D. Zaremba, P. Kaifosh, J. Bowler, M. Ladow, and A. Losonczy. 376 Sublayer-Specific Coding Dynamics during Spatial Navigation and Learning in Hippocampal 377 Area CA1. Neuron, 91:652-665, 2016. doi: 10.1016/j.neuron.2016.06.020. URL 378 http://dx.doi.org/10.1016/j.neuron.2016.06.020.

D. A. Dombeck, A. N. Khabbaz, F. Collman, T. L. Adelman, and D. W. Tank. Imaging large-scale 380 neural activity with cellular resolution in awake, mobile mice. Neuron, 56(1):43-57, oct 2007. ISSN 0896-6273. doi: 10.1016/j.neuron.2007.08.003. URL

http://www.ncbi.nlm.nih.gov/pubmed/17920014http: //www.pubmedcentral.nih.gov/articlerender.fcgi?artid=PMC2268027.

I. Dragatsis and S. Zeitlin. CaMKII?-cre transgene expression and recombination patterns in the 385 mouse brain. genesis, 26(2):133-135, feb 2000. ISSN 1526-954X. doi: 386 10.1002/(SICI)1526-968X(200002)26:2〈133::AID-GENE10〉3.0.CO;2-V. URL 387

[ http://doi.wiley.com/10.1002/ 388 $\{\%\} 28$ SICI $\{\%\} 291526-968 X\{\%\} 28200002\{\%\} 2926\{\%\} 3 A 2\{\%\} 3 C 133\{\%\} 3 A\{\%\} 3 A A I D-G E N E 10\{\%\} 3 E 3.389$ $0 . \mathrm{CO}\{\%\} 3 \mathrm{~B} 2-\mathrm{V}$. 
H. Eichenbaum. On the Integration of Space, Time, and Memory. Neuron, 95(5):1007-1018, aug 391 2017. ISSN 0896-6273. doi: 10.1016/J.NEURON.2017.06.036. URL https: //wWw.sciencedirect.com/science/article/pii/S0896627317305603?via\{\%\}3Dihub. 393

M. S. Fanselow. From contextual fear to a dynamic view of memory systems. Trends Cogn Sci, 14394 (1):7-15, 2010. ISSN 1879-307X (Electronic) 1364-6613 (Linking). doi: 10.1016/j.tics.2009.10.008. 395 URL https://www.ncbi.nlm.nih.gov/pubmed/19939724.

M. Fendt, M. S. Fanselow, and M. Koch. Lesions of the Dorsal Hippocampus Block Trace Fear 397 Conditioned Potentiation of Startle. Behav. Neurosci., 119(3):834-838, 2005. ISSN 1939-0084. 398 doi: 10.1037/0735-7044.119.3.834. URL http://doi.apa.org/getdoi.cfm?doi=10.1037/0735-7044.119.3.834.

J. Friedrich, P. Zhou, and L. Paninski. Fast online deconvolution of calcium imaging data. PLOS ${ }_{401}$ Comput. Biol., 13(3):e1005423, mar 2017. ISSN 1553-7358. doi: 10.1371/journal.pcbi.1005423. ${ }_{402}$ URL http://dx.plos.org/10.1371/journal.pcbi.1005423.

S. Fusi, W. F. Asaad, E. K. Miller, and X.-J. Wang. A neural circuit model of flexible sensorimotor 404 mapping: learning and forgetting on multiple timescales. Neuron, 54(2):319-333, 2007.

B. F. Grewe, J. Grundemann, L. J. Kitch, J. A. Lecoq, J. G. Parker, J. D. Marshall, M. C. Larkin, 406 P. E. Jercog, F. Grenier, J. Z. Li, A. Luthi, and M. J. Schnitzer. Neural ensemble dynamics 407 underlying a long-term associative memory. Nature, 543(7647):670-675, 2017. ISSN 1476-4687 408 (Electronic) 0028-0836 (Linking). doi: 10.1038/nature21682. URL 409 https://www.ncbi.nlm.nih.gov/pubmed/28329757.

J. Grundemann and A. Luthi. Ensemble coding in amygdala circuits for associative learning. Curr 411 Opin Neurobiol, 35:200-6, 2015. ISSN 1873-6882 (Electronic) 0959-4388 (Linking). doi: 412 10.1016/j.conb.2015.10.005. URL https://www.ncbi.nlm.nih.gov/pubmed/26531780. 413

Z. V. Guo, S. A. Hires, N. Li, D. H. O'Connor, T. Komiyama, E. Ophir, D. Huber, C. Bonardi, ${ }_{414}^{4}$ K. Morandell, D. Gutnisky, S. Peron, N. L. Xu, J. Cox, and K. Svoboda. Procedures for 415 behavioral experiments in head-fixed mice. PLoS One, 9(2):e88678, 2014. doi: 416 10.1371/journal.pone.0088678. URL https://www.ncbi.nlm.nih.gov/pubmed/24520413. 417 
P. T. Huerta, L. D. Sun, M. A. Wilson, and S. Tonegawa. Formation of temporal memory requires ${ }_{418}$ NMDA receptors within CA1 pyramidal neurons. Neuron, 25(2):473-480, Feb 2000.

P. Kaifosh, M. Lovett-Barron, G. F. Turi, T. R. Reardon, and A. Losonczy. Septo-hippocampal 420 GABAergic signaling across multiple modalities in awake mice. Nat. Neurosci., 16(9):1182-1184, 421 sep 2013. ISSN 1097-6256. doi: 10.1038/nn.3482. URL http://www.nature.com/articles/nn.3482.

P. Kaifosh, J. D. Zaremba, N. B. Danielson, and A. Losonczy. SIMA: Python software for analysis 424 of dynamic fluorescence imaging data. Front. Neuroinform., 8:80, sep 2014. ISSN 1662-5196. doi: 425 10.3389/fninf.2014.00080. URL

J. Kaminski, S. Sullivan, J. M. Chung, I. B. Ross, A. N. Mamelak, and U. Rutishauser. Persistently ${ }_{428}$ active neurons in human medial frontal and medial temporal lobe support working memory. Nat ${ }_{429}$ Neurosci, 20(4):590-601, 2017. doi: 10.1038/nn.4509. URL http://www.ncbi.nlm.nih.gov/pubmed/28218914.

T. Kitamura, C. J. Macdonald, and S. Tonegawa. Entorhinal-hippocampal neuronal circuits bridge ${ }^{436}$ temporally discontiguous events. Learn. Mem., 22(9):438-43, sep 2015. ISSN 1549-5485. doi: ${ }^{437}$ 10.1101/lm.038687.115. URL

http://learnmem.cshlp.org/lookup/doi/10.1101/lm.038687.115http:

H. Kuhn. The hungarian method for the assignment problem. Naval Research Logistics Quarterly, ${ }_{442}$ 2:83-97, 1955.

J. J. Letzkus, S. B. Wolff, and A. Luthi. Disinhibition, a circuit mechanism for associative learning ${ }_{444}$ 
and memory. Neuron, 88(2):264-76, 2015. ISSN 1097-4199 (Electronic) 0896-6273 (Linking). doi: ${ }_{445}$ 10.1016/j.neuron.2015.09.024. URL https://www.ncbi.nlm.nih.gov/pubmed/26494276. 446

X. Liu, S. Ramirez, P. T. Pang, C. B. Puryear, A. Govindarajan, K. Deisseroth, and S. Tonegawa. ${ }^{447}$ Optogenetic stimulation of a hippocampal engram activates fear memory recall. Nature, $484 \quad 448$ (7394):381-5, 2012. doi: 10.1038/nature11028. URL 449 https://www.ncbi.nlm.nih.gov/pubmed/22441246. 450

M. Lovett-Barron, P. Kaifosh, M. A. Kheirbek, N. Danielson, J. D. Zaremba, T. R. Reardon, G. F. ${ }^{451}$ Turi, R. Hen, B. V. Zemelman, and A. Losonczy. Dendritic Inhibition in the Hippocampus 452 Supports Fear Learning. Science, 343(6173), 2014. URL ${ }_{453}$ http://science.sciencemag.org/content/343/6173/857. 454

C. MacDonald, K. Lepage, U. Eden, and H. Eichenbaum. Hippocampal "Time Cells" Bridge the ${ }_{455}$ Gap in Memory for Discontiguous Events. Neuron, 71(4):737-749, aug 2011. ISSN 0896-6273. 456 doi: 10.1016/J.NEURON.2011.07.012. URL 457 https://www.sciencedirect.com/science/article/pii/S089662731100609X. 458

C. J. MacDonald, S. Carrow, R. Place, and H. Eichenbaum. Distinct hippocampal time cell ${ }^{459}$ sequences represent odor memories in immobilized rats. J Neurosci, 33(36):14607-16, 2013. doi: 460 10.1523/JNEUROSCI.1537-13.2013. URL http://www.ncbi.nlm.nih.gov/pubmed/24005311. 461

S. A. Maddox, J. Hartmann, R. A. Ross, and K. J. Ressler. Deconstructing the gestalt: ${ }^{462}$ Mechanisms of fear, threat, and trauma memory encoding. Neuron, 102(1):60-74, 2019. ISSN ${ }_{463}$ 1097-4199 (Electronic) 0896-6273 (Linking). doi: 10.1016/j.neuron.2019.03.017. URL 464 https://www.ncbi.nlm.nih.gov/pubmed/30946827. 465

A. Malvache, S. Reichinnek, V. Villette, C. Haimerl, and R. Cossart. Awake hippocampal 466 reactivations project onto orthogonal neuronal assemblies. Science, 353(6305):1280-3, 2016. ISSN 467 1095-9203 (Electronic) 0036-8075 (Linking). doi: 10.1126/science.aaf3319. URL ${ }_{468}$ https://www.ncbi.nlm.nih.gov/pubmed/27634534. 469

S. Maren, K. L. Phan, and I. Liberzon. The contextual brain: implications for fear conditioning, 470 extinction and psychopathology. Nat Rev Neurosci, 14(6):417-28, 2013. ISSN 1471-0048 471 
(Electronic) 1471-003X (Linking). doi: 10.1038/nrn3492. URL

G. Mongillo, O. Barak, and M. Tsodyks. Synaptic theory of working memory. Science, 319(5869): 474 1543-6, 2008. doi: 10.1126/science.1150769. URL

M. Pachitariu, C. Stringer, M. Dipoppa, S. Schröder, L. F. Rossi, H. Dalgleish, M. Carandini, and ${ }_{477}$ K. D. Harris. Suite2p: beyond 10,000 neurons with standard two-photon microscopy. bioRxiv, ${ }_{478}$ 2017. doi: https://doi.org/10.1101/061507.

E. Pastalkova, V. Itskov, A. Amarasingham, and G. Buzsáki. Internally generated cell assembly 480 sequences in the rat hippocampus. Science, 321(5894):1322-7, sep 2008. ISSN 1095-9203. doi: ${ }^{481}$ प 10.1126/science.1159775. URL http://www.ncbi.nlm.nih.gov/pubmed/18772431http: //wWw.pubmedcentral.nih.gov/articlerender.fcgi?artid=PMC2570043.

F. Pedregosa, G. Varoquaux, A. Gramfort, V. Michel, B. Thirion, O. Grisel, M. Blondel,

P. Prettenhofer, R. Weiss, V. Dubourg, J. Vanderplas, A. Passos, D. Cournapeau, M. Brucher, ${ }_{485}$ M. Perrot, and É. Duchesnay. Scikit-learn: Machine Learning in Python. J. Mach. Learn. Res., ${ }^{486}$ 12(Oct):2825-2830, 2011. ISSN ISSN 1533-7928. URL http://www.jmlr.org/papers/v12/pedregosa11a.html. differentially mediate trace and contextual fear conditioning. Hippocampus, 15(5):665-674, 2005 . 490 ISSN 1050-9631. doi: 10.1002/hipo.20088. URL http://doi.wiley.com/10.1002/hipo.20088. 
A. J. Rashid, C. Yan, V. Mercaldo, H. L. Hsiang, S. Park, C. J. Cole, A. De Cristofaro, J. Yu, ${ }^{499}$ C. Ramakrishnan, S. Y. Lee, K. Deisseroth, P. W. Frankland, and S. A. Josselyn. Competition 500 between engrams influences fear memory formation and recall. Science, 353(6297):383-7, $2016 . \quad 501$ ISSN 1095-9203 (Electronic) 0036-8075 (Linking). doi: 10.1126/science.aaf0594. URL 502 https://www.ncbi.nlm.nih.gov/pubmed/27463673.

J. D. Raybuck and K. M. Lattal. Bridging the interval: Theory and neurobiology of trace conditioning. Behav. Processes, 101:103-111, jan 2014. ISSN 0376-6357. doi:

N. T. Robinson, J. B. Priestley, J. W. Rueckemann, A. D. Garcia, V. A. Smeglin, F. A. Marino, primate amygdala and prefrontal cortex. Neuron, 87:869-81, 2015.

A. Sellami, A. S. Al Abed, L. Brayda-Bruno, N. Etchamendy, S. Valério, M. Oulé, L. Pantaléon, 
K. Takehara-Nishiuchi and B. L. McNaughton. Spontaneous changes of neocortical code for ${ }_{527}$ associative memory during consolidation. Science, 322(5903):960-3, 2008. doi: 528 10.1126/science.1161299. URL http://www.ncbi.nlm.nih.gov/pubmed/18988855. 529

K. Z. Tanaka, H. He, A. Tomar, K. Niisato, A. J. Y. Huang, and T. J. McHugh. The hippocampal 530 engram maps experience but not place. Science, 361(6400):392-397, 2018. doi: 531 $10.1126 /$ science.aat5397. 532

K. K. Tayler, K. Z. Tanaka, L. G. Reijmers, and B. J. Wiltgen. Reactivation of neural ensembles 533 during the retrieval of recent and remote memory. Curr Biol, 23(2):99-106, 2013. doi: 534 10.1016/j.cub.2012.11.019. URL https://www.ncbi.nlm.nih.gov/pubmed/23246402. 535

G. P. Urcelay and R. R. Miller. The functions of contexts in associative learning. Behav Processes, 536 104:2-12, 2014. ISSN 1872-8308 (Electronic) 0376-6357 (Linking). doi: 537 10.1016/j.beproc.2014.02.008. URL https://www.ncbi.nlm.nih.gov/pubmed/24614400. 538

G. Vetere, J. W. Kenney, L. M. Tran, F. Xia, P. E. Steadman, J. Parkinson, S. A. Josselyn, and 539 P. W. Frankland. Chemogenetic interrogation of a brain-wide fear memory network in mice. 540 Neuron, 94(2):363-374 e4, 2017. doi: 10.1016/j.neuron.2017.03.037. URL 541 https://www.ncbi.nlm.nih.gov/pubmed/28426969. 542

Y. Wang, S. Romani, B. Lustig, A. Leonardo, and E. Pastalkova. Theta sequences are essential for 543 internally generated hippocampal firing fields. Nat. Neurosci., 18(2):282-288, feb 2015. ISSN 544 1097-6256. doi: 10.1038/nn.3904. URL http://www.nature.com/articles/nn.3904. 545

J. Yamamoto and S. Tonegawa. Direct medial entorhinal cortex input to hippocampal cal is crucial 546 for extended quiet awake replay. Neuron, 96:217-27, 2017.

A. P. Yiu, V. Mercaldo, C. Yan, B. Richards, A. J. Rashid, H. L. Hsiang, J. Pressey, V. Mahadevan, 548 M. M. Tran, S. A. Kushner, M. A. Woodin, P. W. Frankland, and S. A. Josselyn. Neurons are ${ }_{549}$ recruited to a memory trace based on relative neuronal excitability immediately before training. 550 Neuron, 83(3):722-35, 2014. ISSN 1097-4199 (Electronic) 0896-6273 (Linking). doi: 551 10.1016/j.neuron.2014.07.017. URL https://www.ncbi.nlm.nih.gov/pubmed/25102562. 552 


\section{Materials and Methods}

\section{Behavior and Imaging}

Mice and viruses All experiments were conducted in accordance with the NIH guidelines and 555 with the approval of the Columbia University Institutional Animal Care and Use Committee. 556 Optogenetic experiments were performed with adult (8-14 weeks) male and female C57/Bl6 mice 557 (Jackson Laboratory) injected bilaterally (see below) with either recombinant adeno-associated 558 virus (rAAV) expressing ArchT (rAAV1/2-Syn-ArchT) or tdTomato control protein 559 (rAAV1/2-Syn-tdTom), under the Synapsin promoter. These viruses were the generous gift of Dr. 560 Boris Zemelman. Imaging experiments were performed with adult (8-16 weeks) male and female 561 transgenic CaMKII $\alpha$-Cre mice on a C57/Bl6 background, where Cre is predominantly expressed in 562 pyramidal neurons (R4Ag11 line, Dragatsis and Zeitlin 2000]; Jackson Laboratory, Stock No: 563 027400). Cre-dependent recombinant adeno-associated virus (rAAV) expressing GCaMP6f 564 (rAAV1-Syn-Flex-GCaMP6f-WPRE-SV40, Penn Vector Core) was used for labeling of pyramidal 565 neurons.

Surgical procedure Viral delivery to hippocampal area CA1 and implantation of headposts, $\quad{ }_{567}$ ¡ptical fibers, and imaging cannulae were as described previously Kaifosh et al., 2013, Kheirbek 568 et al. 2013, Lovett-Barron et al., 2014. Briefly, viruses were delivered to dorsal CA1 by stereotactically injecting $50 \mathrm{~nL}(10 \mathrm{~nL}$ pulses) of rAAVs at three dorsoventral locations using a 570 Nanoject syringe (-2.3 mm AP; -1.5 mm ML; -0.9, -1.05 and -1.2 mm DV relative to bregma). For 571 head-fixed optogenetic experiments, mice were chronically implanted with bilateral optical fiber 572 cannulae above the CA1 injection sites after virus delivery Lovett-Barron et al., 2014, Kheirbek 573 et al. 2013]. A stainless steel headpost was then fixed to the skull Kaifosh et al., 2013]. The 574 cannula, headpost, and any exposed skull were secured and covered with black grip cement to block 575 light from the implanted optical fibers. For imaging experiments, mice were allowed to recover in 576 their home cage for 3 days following virus delivery procedures. They were then surgically implanted 577 with a steel headpost along with an imaging window (diameter, $3.0 \mathrm{~mm}$; height, $1.5 \mathrm{~mm}$ ) over the 578 left dorsal hippocampus. Imaging cannulae were constructed by adhering (Narland optical adhesive) 579 
a $3 \mathrm{~mm}$ glass coverslip (64-0720, Warner) to a cylindrical steel cannula. The imaging window 580 surgical procedure was performed as detailed previously Kaifosh et al., 2013, Lovett-Barron et al., 581 2014]. For all surgeries, analgesia was continued for 3 days postoperatively.

Behavioral apparatus We adopted our previously described Kaifosh et al., 2013, Lovett-Barron et al., 2014 head-fixed system for combining 2-photon imaging with 584 microcontroller-driven (Arduino) stimulus presentation and behavioral read-out. To maintain 585 immobility and constrain neural activity related to locomotion MacDonald et al. 2013, mice were 586 head-fixed in a body tube chamber Guo et al. 2014]. The chamber was lined with textured fabric 587 that was interchanged between trials to prevent contextual conditioning. Tones were presented via 588 nearby speakers and air-puffs delivered by actuating a solenoid valve, which gated airflow from a 589 compressed air tank to a pipette tip pointed at the mouse's snout. Water reward delivery during 590 licking behavior was gated by another solenoid valve in response to tongue contact with a metal 591 water port coupled to a capacitive sensor. Electrical signals encoding mouse behavior and stimulus 592 presentation were collected with an analog-to-digital converter, which was synchronized with either 593 optogenetic laser delivery or 2-photon image acquisition by a common trigger pulse.

Head-fixed trace fear conditioning Starting 3-7 days after surgical implantation, mice were 595 habituated to handling and head-fixation as previously described Kaifosh et al. 2013. Lovett-Barron et al., 2014, Guo et al., 2014]. Within 3 days, mice could undergo up to an hour of 597 head-fixation on the behavioral apparatus while remaining calm and alert. They were then water 598 deprived to 85-90\% of their starting body weight and trained to lick operantly for small-volume 599 water rewards ( $500 \mathrm{~nL} /$ lick) while head-fixed. Before undergoing experimental paradigms, mice $\quad{ }_{600}$ were required to maintain consistent licking for multiple (6-12) 60 second trials per day while 601 maintaining their body weight between 85-90\% of starting weight. 602

For optogenetic experiments, we utilized our previously described head-fixed 'trace' fear ${ }_{603}$ conditioning paradigm Kaifosh et al., 2013]. Briefly, we paired a 20 second auditory conditioned 604 stimulus (CS, either $10 \mathrm{kHz}$ constant tone or $2 \mathrm{kHz}$ tone pulsed at 1Hz) with air-puffs 605 (unconditioned stimulus, US; $200 \mathrm{~ms}, 5$ puffs at $1 \mathrm{~Hz}$ ), separated by a 15 second stimulus-free 'trace' ${ }_{606}$ 
period. During each conditioning trial, we recorded licking from mice over a 55 second period: $10 \quad 607$ second pre-CS, 20 second CS, 15 second trace, and 5 second US. Mice were conditioned across trials 608 spaced throughout three consecutive days. On each trial, we used suppression of licking during the ${ }_{609}$ tone, normalized to licking during the 10 second pre-CS period, as a measure of conditioned fear. ${ }_{610}$ We changed the fabric material in the behavioral chamber between every trial to prevent contextual ${ }_{611}$ fear conditioning [Kaifosh et al., 2013, Lovett-Barron et al., 2014].

For 2-photon imaging experiments, we expanded our behavioral paradigm to a differential ${ }_{613}$ learning assay using the 2 different auditory cues above as either a CS+ or CS- (where only CS+ is ${ }_{614}$ paired with the aversive US). We randomized the assignment of CS+ and CS- tones across mice. ${ }_{615}$ Prior to the introduction of US-paired conditioning trials, we obtained multiple trials of behavioral 616 responses (10-15 trials; "Pre-Learning") to each CS cue presented alone in pseudorandom order ${ }_{617}$ over 3 days. This helped to confirm that subsequent lick suppression was not due to 618 'pseudoconditioning' or stimulus novelty effects Burman et al. 2014. We then subjected mice to ${ }_{619}$ our 3-day conditioning protocol with US-pairing as above, but with alternation between CS+ and ${ }_{620}$ CS- trials ('Learning'). Finally, over another 3 days, we collected additional trials ( 20-25 of each ${ }^{621}$ CS presented in pseudo-random order, 'Post-Learning') with continued US reinforcement on CS+ ${ }_{622}$ trials. During Pre-Learning and Post-Learning trials, contextual cues, consisting of the chamber ${ }_{623}$ fabric material and a background odor of either $70 \%$ ethanol or $2 \%$ acetic acid, were randomly ${ }_{624}$ changed across trials.

Head-fixed optogenetics $200 \mu \mathrm{m}$ core, 0.37 numerical aperture (NA) multimode optical fibers 626 were constructed as previously detailed [Kheirbek et al. 2013]. A splitter patch cable (Thorlabs) ${ }_{627}$ was used to couple bilaterally implanted optical fibers to a $532 \mathrm{~nm}$ laser (50 mW, OptoEngine) for ${ }_{628}$ ArchT activation while mice were head-fixed. All cables/connections were shielded to prevent light ${ }_{629}$ leak from laser stimuli and matching-color ambient LED illumination was continuously provided in 630 the behavioral apparatus so as to prevent the laser activation from serving as a visual cue. After ${ }_{631}$ the 10 second pre-CS period on each trace fear conditioning trial, $10 \mathrm{~mW}$ of laser light was 632 continuously delivered through each optical fiber for the entire CS-trace-US sequence. 633 Experimenters were blinded to subject viral injections. After data collection, mice were processed ${ }_{634}$ 
for histology and recovery of optical fibers. Subjects were excluded from the study if the implant ${ }_{635}$ entered the hippocampus, if viral infection was not complete in dorsal CA1, or if there were signs of ${ }_{636}$ damage to the optical fiber that could have compromised intracranial light delivery.

2-photon microscopy For imaging experiments, mice were habituated to the imaging apparatus 638 (e.g. microscope/objective, laser, sounds of resonant scanner and shutters) during the training ${ }_{639}$ period. All imaging was conducted using a 2-photon $8 \mathrm{kHz}$ resonant scanner (Bruker). As described ${ }_{640}$ in Danielson et al. 2016], we coupled a piezoelectric crystal to the objective (Nikon 40x NIR water ${ }_{641}$ immersion, $0.8 \mathrm{NA}, 3.5 \mathrm{~mm}$ working distance), allowing for rapid displacement of the imaging plane ${ }_{642}$ in the $\mathrm{z}$ dimension, which permitted simultaneous data collection from CA1 neurons in 2 different ${ }_{643}$ optical sections. To align the CA1 pyramidal layer with the horizontal two-photon imaging plane, ${ }_{644}$ we adjusted the angle of the mouse's head using two goniometers ( $\pm 10^{\circ}$ range, Edmund Optics). ${ }_{645}$ For excitation, we used a $920 \mathrm{~nm}$ laser (50-100 $\mathrm{mW}$ at objective back aperture, Coherent). Green ${ }_{646}$ (GCaMP6f) fluorescence was collected through an emission cube filter set (HQ525/70 m-2p) to a ${ }_{647}$ GaAsP photomultiplier tube detector (Hamamatsu, 7422P-40). A custom dual stage preamp (1.4 x 648 $105 \mathrm{~dB}$, Bruker) was used to amplify signals prior to digitization. All experiments were performed 649 at 1.5-2x digital zoom, covering $\sim 150-200 \mathrm{~mm} \times 150-200 \mathrm{~mm}$ in each imaging plane. 2-plane images 650 (512 x 512 pixels each) were separated by $20 \mu \mathrm{m}$ in the optical axis and acquired at $\sim 8 \mathrm{~Hz}$ given a 651 $30 \mathrm{~ms}$ settling time of the piezo z-device.

Image preprocessing All imaging data were pre-processed using the SIMA software package ${ }_{653}$ Kaifosh et al. 2014. Motion correction was performed using a modified 2D hidden Markov model ${ }_{654}$ Dombeck et al. 2007, Kaifosh et al., 2013 in which the model was re-initialized on each plane in 655 order to account for the settling time of the piezo. In cases where motion artifacts were not 656 adequately corrected, the affected data were discarded from further analysis. We used the Suite2p ${ }_{657}$ software package [Pachitariu et al., 2017] to identify spatial masks corresponding to neural region of 658 interest (ROIs) and extract associated fluorescence signal within these spatial footprints, correcting 659 for cross-ROI and neuropil contamination. Identified ROIs were curated post-hoc using the Suite2p 660 graphical interface to exclude non-somatic components. 
ROI detection with Suite2p is inherently activity-dependent, and so for each session, we detected 662 only a subset of neurons that were physically present in the FOV. Once signals were extracted for ${ }_{663}$ all sessions, we registered ROIs across each session as follows. We first chose the session with the ${ }_{664}$ largest number of detected neurons as the reference session, and then computed an affine transform 665 between the time-averaged FOV of all other sessions to the reference. Transforms were visually ${ }_{666}$ inspected to verify accuracy. Using these transforms, we processed each session serially to register ${ }_{667}$ ROIs to a common neural pool across sessions. For a given session (referred to now as the current ${ }_{668}$ session), the calculated FOV transform was applied to all ROI masks to map them to the reference ${ }_{669}$ session coordinates. We calculated a distance matrix (using Jaccard similarity) that quantified the 670 spatial overlap between all pairs of reference and current session ROIs. We then applied the ${ }^{671}$ Hungarian algorithm Kuhn, 1955 to identify the optimal pairs of reference and current ROIs. All 672 pairs with a Jaccard distance below 0.5 were automatically accepted as the same ROI. For the 673 remaining unpaired current ROIs, pairs were manually curated via an IPython notebook, which 674 allowed the user to select the appropriate reference ROI to pair or enter the current ROI as a new 675 ROI (i.e., not in the reference pool). Any current ROIs whose centroids that were more than $50 \quad 676$ pixels away from an unpaired reference ROI were automatically entered as new ROIs, to accelerate 677 the curation. Once all ROIs for the current session were processed (either paired with a reference 678 ROI or labeled as new), the new ROIs were appended to the reference list. The remaining sessions 679 were then processed serially in the same fashion, where the reference ROI list is augmented on each 680 step to include additional ROIs that were not presented in any previously processed session. Once 681 all sessions were processed, this process yielded a complete list of reference ROIs and their identity 682 in each individual session. As a final step, the reference ROIs were warped back to the FOVs of ${ }_{683}$ each individual session via affine transform and ROIs that fell outside the boundaries of any session ${ }_{684}$ FOV were discarded, so that all analyzed neurons were physically in view for all sessions. Inbound 685 reference ROIs that were not present in individual sessions were assumed to be silent in subsequent ${ }_{686}$ $\begin{array}{ll}\text { analysis. } & 687\end{array}$ 
Event detection All fluorescence traces were deconvolved to detect putative spike events, using ${ }_{689}$ the OASIS implementation of the fast non-negative deconvolution algorithm [Friedrich et al., 2017]. 690 Following spike inferencing, we discarded any events whose energy was below 4 median absolute ${ }_{691}$ deviations of the raw trace. This avoided including small events within the range of the noise, 692 which could artificially inflate activity rates and correlations between neurons.

Given the dominant sparsity of activity, we then discretized ROI trace to indicate whether an 694 event was present in each frame. Trials for each experiment were collected over the span of several 695 days. Consequently, we found that discretization was necessary to prevent variations in imaging 696 system parameters from exerting undue influences on the analysis, as this could introduce arbitrary ${ }_{697}$ variance in the scale of calcium events across sessions.

Decoding All classifiers were support vector machines (SVM) with a linear kernel, using the ${ }_{699}$ implementation in scikit-learn Pedregosa et al. 2011]. For cross-validation, data were randomly 700 divided into non-overlapping training and test sets (75/25\% split). This procedure was repeated 701 100 times for each classifier with random training/test subdivisions and reported as the average 702 across cross-validation folds. Trials were balanced by subsampling the overrepresented class, and all 703 decoding results were compared against a null distribution built by repeating the analyses on 704 appropriately shuffled surrogate data, which controlled for the effects of finite sampling. This is 705 particularly important for fear learning paradigms such as ours, where trial counts are very limited. 706

Decoding elapsed time We designed a decoder to predict the elapsed time during each trial, in 707 order to assess whether there were consistent temporal dynamics in the neural data during the 708 experiment, such as sequences of time cells. Time decoding was done separately for CS+ and CS- 709 trials, and for Pre- and Post-Learning trial blocks, to assess differences between cues and over 710 learning. We analyzed data during both the tone and trace period, for a total of 35 seconds on each 711 trial. We averaged each neuron's activity in non-overlapping 2.5 second bins, so that each trial was 712 described by a sequence of 14 population vectors of activity in time. 
Our time decoder uses a 1 vs 1 approach through an ensemble of linear classifiers. For each pair 714 of time bins in the experiment, we train a separate classifier to distinguish between population $\quad 715$ vectors of activity that came from those two time bins. For comparing 14 time bins, this results in 716 a set of 91 binary classifiers trained on all unique time comparisons Bakhurin et al., 2017, Cueva 717 et al. 2019]. We first evaluated the performance of the individual classifiers by testing their ability 718 to correctly label time bins from held out test trials, where in this analysis each classifier is tested 719 only on time bins from the trial times that it was trained to discriminate. This result is presented 720 as a matrix in Figure 2, and demonstrates the linear separability of any two points in time during 721 the task. We then used the decoder to perform a multi-class time prediction analysis. Here each ${ }_{722}$ population vector in a held-out test trial is presented to all classifiers, which "vote" on what time ${ }_{723}$ bin the data came from. We take the decoded time to be the time bin with the plurality of votes, ${ }^{724}$ and repeat this procedure across all time bins in each test trial to decode the passage of time. ${ }_{725}$

For all time decoding analyses, we compare the classification accuracy or prediction error to a 726 null distribution, which we calculate by repeating these analyses on 1000 surrogate data sets, where ${ }_{727}$ for each trial independently, we randomly permute the order of the time bins. This destroys any 728 consistent temporal information across trials, while preserving the average firing rates and 729 correlations between neurons within each trial.

Decoding task variables from instantaneous firing rates We also used a population 731 decoding approach to assess the times in the experiment during which there was significant 732 information about the stimulus identity in the neural data. On each trial, we averaged the activity 733 of each neuron in non-overlapping $1 \mathrm{sec}$ bins. We then trained a separate linear classifier on each 734 time bin to predict whether the population vector came from a CS+ or CS- trial. Classifiers were 735 cross-validated as above on held-out test data. We similarly compared the classification accuracy 736 for trial information to a null distribution, where here we repeated the decoding analysis on $1000 \quad 737$ surrogate data sets where the CS trial label was randomly shuffled.

Decoding from average firing rates We similarly assessed our ability to decode task 739 information from the average firing rates of the neurons within a set trial period on each trial. This 740 
procedure is identical to the one outlined above, and we used it to assess our ability to decoder the 741 CS identity during the Pre-CS (-5 to $0 \mathrm{sec}$ ), CS (0 to $20 \mathrm{sec}$ ), Trace (20 to $35 \mathrm{sec}$ ), US (35 to 40742 sec), and Post-US (40 to $170 \mathrm{sec}$ ) periods of the trial (Fig. 4 4 ), as well as during the CS and trace ${ }_{743}$ periods combined ( 0 to $35 \mathrm{sec}, 4 \mathrm{~A})$.

We additionally assessed how decoders learned at one time period in the task generalize to 745 activity observed in other time periods, by constructing a cross-time period decoding analysis. Here 746 on each cross-validation fold, we trained different decoders to predict CS identity from the activity 747 during each trial period separately. Then on the held-out test data, we tested each decoder not only 748 on the activity from the time period on which it was trained, but on the activity of all other time 749 periods as well (e.g. train on CS, test on trace). The result is a matrix of pairwise trial period 750 comparisons, where the columns indicate the time period used for training the classifier and the row 751 indicate the time period for testing. These comparisons are not necessarily symmetric (e.g., we find 752 that CS-period decoders can be used to predict the cue when tested on trace-period activity better 753 $\begin{array}{ll}\text { than the reverse). } & 754\end{array}$

Probabilistic decoding We additionally assessed our ability to decode the CS identity using a 755 Bayesian approach (see S3). The cross-validation and significance methods were identical to the 756 SVM analysis described previously. For every fold, we used a multinomial naïve Bayes classifier to 757 predict the CS cue from the event counts in a given time bin.

Selectivity index analysis To assess CS-selectivity at the level of single neurons, we computed 759 a selectivity index as:

$$
S I=\frac{f_{+}-f_{-}}{f_{+}+f_{-}}
$$

where $f_{+}$and $f_{-}$are the average activity of the neuron from 0-35 sec on CS+ and CS- trials 761 respectively. This yields an index bounded between +1 (all activity during CS + trials) and -1 (all 762 activity during CS- trial). Similar to the decoding analysis, we compared this selectivity index to 763 those calculated from 1000 surrogate datasets where the trial type labels were randomly shuffled, $\quad 764$ 
which controlled for spurious firing rate differences attributable to small numbers of trials. We 765 computed these scores separately for Pre- and Post-Learning trials, and quantified the fraction of 766 cells active during that trial type which showed significant CS-selectivity, determined by calculating 767 a p-value from the observed SI relative to its shuffle distribution. For the regression to population 768 decoder weights shown in Fig. $4 \mathrm{E}$, we z-scored each SI relative to its shuffle distribution's mean and 769 standard deviation.

Ensemble overlap analysis We measured the similarity in the active set of neurons between a 771 pair of trials by computing the Jaccard similarity index, which for two sets is given by the size of 772 the set intersection divided by the size of the set union (Fig. 5). Intuitively, if the sets of active 773 neurons overlap completely, the set intersection and union are equal and the index is 1, while if 774 trials recruit orthogonal sets of neurons, the intersection and thus the index are 0. This metric is 775 biased by the fraction of active neurons on a given trial; if two trials randomly recruit $50 \%$ of 776 neurons, the Jaccard similarity will tend to be higher than if they randomly recruited $10 \%$ of 777 neurons, as the former will tend to have more overlapping elements purely by chance. To control for 778 differences in activity rates across trials, we generated 1000 surrogate scores for each trial pair 779 where we recomputed the index between two binary vectors whose elements were randomly assigned 780 as active or inactive to match the fraction of active neurons in the real trials. The observed index 781 was then z-normalized relative to this distribution, to quantify the population similarity beyond 782 that expected from random recruitment of the same number of neurons. 783

Sequence score For analysis of neural sequences during initial Learning trials, we detected the 784 latency to peak firing rate for each neuron that was active on at least 2 Learning trials. We 785 compared this firing order between all Learning trial pairs via Spearman's rank correlation, and 786 assigned a sequence score as the average pairwise rank correlation between trials. This analysis was 787 done separately for CS+ and CS- trials. To assess significance, sequence scores were compared to 788 those calculated from 1000 surrogate datasets, where for each neuron, its activity trace was 789 randomly permuted on each trial independently to randomize the temporal ordering between cells. 790 
A

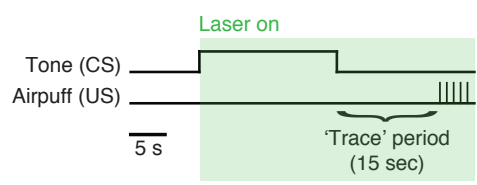

C

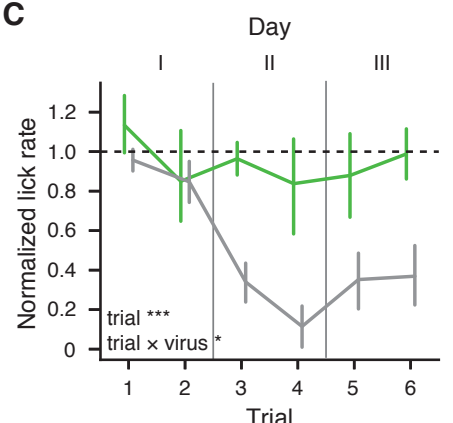

B

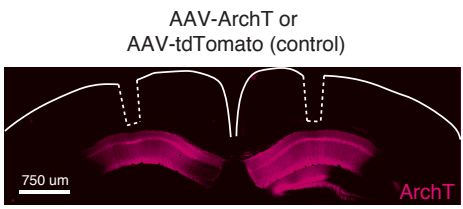

D

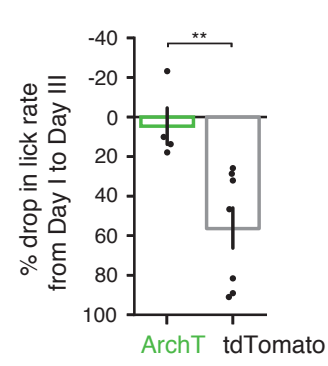

$\mathbf{E}$

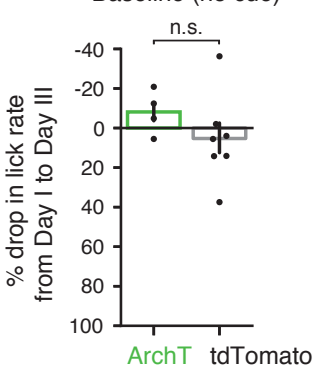

Figure S1. Head-fixed auditory trace fear conditioning is dependent on dorsal CA1 activity. A: Schematic of behavior protocol. On each trial, animals are exposed to a 20 second auditory tone (CS) followed by a 15 second trace period. Following the trace period, airpuffs are delivered to the animal's snout (US). Animals are water deprived and operant rewards are available through each trial. Learned fear is measured via suppression of the licking response. On all conditioning trials, laser stimulation is delivered throughout the tone, trace, and airpuff periods. Animals underwent two conditioning trials per day over the course of 3 days. A baseline trial of the same duration was collected prior to each day's conditioning trials, where reward was available without presentation of the CS or US. B: Left: schematic of optic fiber implants. Mice received bilateral injections in dorsal CA1, with either rAAV1/2-Syn-ArchT or rAAV1/2-Syn-tdTom, to express inhibitory opsin or control fluorescent protein constructs, respectively. Optic fiber implants were then positioned above the dorsal hippocampus in each hemisphere. Right: post hoc confirmation of viral expression and optic fiber placement (dotted lines) in an example mouse. C: Normalized licking behavior of ArchT and tdTomato mice in response to the CS cue for all US-paired trials. Laser stimulation prevents learning only in the ArchT group (linear mixed effect model with fixed effects of virus and trial number, and random effect of mouse, significant effects are inset). D: Summary of behavioral impact of CA1 inhibition, calculated as the \% change in normalized lick rate from day I to III (paired t-test). E: As in D, but calculated from the baseline (no CS/US presentations) trials on day I and III. Licking behavior is not affected by optogenetic manipulations in the absence of task stimuli. Data are presented as mean \pm s.e.m. ${ }^{*} p<0.05,{ }^{* *} p<0.01,{ }^{* * *} p<0.001$ 

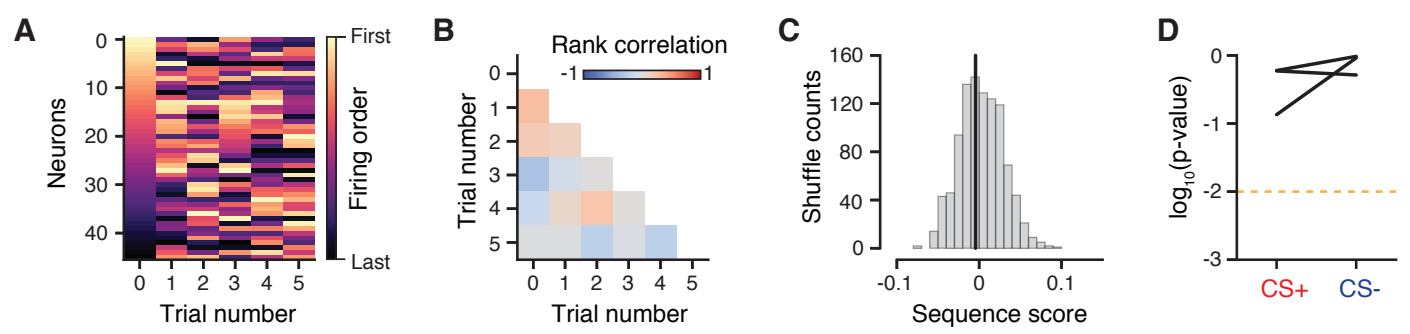

Figure S2. Sequential activity is not reliable during initial learning trials. A: Neural sequences during the initial Learning trials, for an example mouse during CS+ trials. Each trial shows the order of neurons' peak firing time, sorted by firing order on the first trial. Only neurons that were active in at least 2 learning trials are shown. B: Rank correlation of peak firing rate times between Learning trials, for the example mouse shown in A. C: Sequence score (average pairwise trial correlation) for B. Grey: null distribution of sequence scores generated by randomly shuffling neuron firing times on each trial. D: Summary of learning trial sequence scores for all mice. Neural sequences are not significantly different from random across trials for either trial type. 


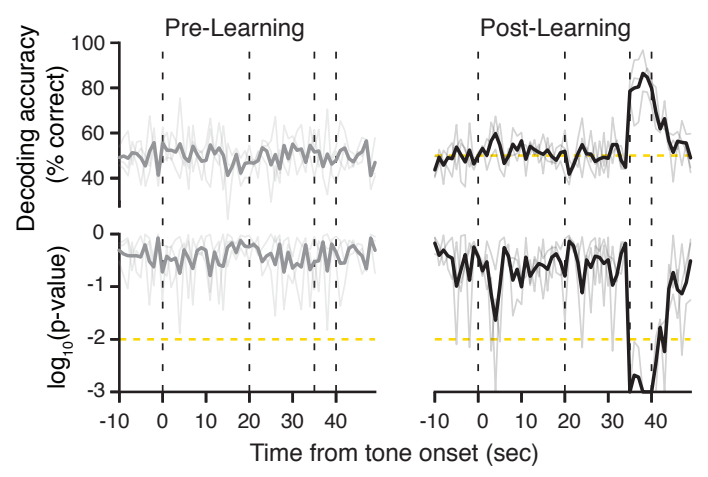

Figure S3. Bayesian decoding of CS identity. We repeated the analysis of decoding accuracy for CS identity shown in Figure 3, here using a naive Bayesian classifier rather than the linear SVM used throughout the paper. The results remained unchanged for both Pre- and Post-Learning trials. 